\title{
FINITE DIMENSIONAL REDUCTION FOR THE POSITIVITY OF SOME SECOND SHAPE DERIVATIVES *
}

\author{
ANTOINE HENROT ${ }^{\dagger}$, MICHEL PIERRE ${ }^{\ddagger}$, AND MOUNIR RIHANI§
}

\begin{abstract}
We study the positivity of the second shape derivative around an equilibrium for a functional defined on exterior domains in the plane and which involves the perimeter of the domains and their Dirichlet energy under volume constraint. We prove that small analytic perturbations of circles may be stable or not, depending on the positivity of a simple and explicit two-variable quadratic form. The approach is general and involves a numerical criterion of independent interest for the positivity of a quadratic form on a given hyperplane.
\end{abstract}

1. Introduction. Our goal is to develop general methods to study the stability of equilibrium shapes for some functionals like those arising in the 2-dimensional modeling of the so-called "exterior shaping problem". "Exterior" means here that the considered variable shapes are "exterior domains", that is, the complement of compact sets in $\mathbb{R}^{2}$.

By equilibrium shape, we mean a regular exterior open subset $\Omega^{*}$ of $\mathbb{R}^{2}$ where the first shape derivative vanishes. By stability, we mean that the second derivative with respect to the shape is strictly positive at $\Omega^{*}$. It is classical that, for regular shapes, the first and second derivatives are respectively linear and quadratic forms on a space of functions from the boundary $\Gamma^{*}$ of $\Omega^{*}$ into $\mathbb{R}$.

We are mainly interested in the case where the measure of the complement of the shapes is prescribed. Therefore, the question concerns the positivity of the second derivative of the full lagrangian (the initial functional together with a Lagrange multiplier term) on the hyperplane tangent to the constraint of prescribed measure. Part of our contribution here is to develop some general tools to do it. In this direction, we provide a useful numerical criterion for checking the positivity of quadratic forms having exactly one negative eigenvalue. Besides its application to our specific functionals, it is illustrated by other examples.

As a good illustration of the functional we consider here, we may think to the shape of a liquid metal confined in an electromagnetic field. The domain occupied by the liquid is a compact set whose complement is an "exterior" open set $\Omega$. The Lebesgue measure of the complement ${ }^{c} \Omega$ of $\Omega$ is prescribed, since it is exactly the domain occupied by the liquid. For a good stability, the equilibrium configuration of the liquid should be a local minimum for the total energy.

According to a natural magnetostatic model, the electromagnetic part of the energy of the system is given by the Dirichlet energy

$$
J(\Omega)=\frac{1}{2} \int_{\Omega}\left|\nabla u_{\Omega}\right|^{2}-\int_{\Omega} f u_{\Omega}
$$

where $f$ is the density of the imposed alternative currents and where $u_{\Omega}$ denotes the

\footnotetext{
* Received August 14, 2003; accepted for publication October 30, 2003.

${ }^{\dagger}$ Ecole des Mines et Institut Elie Cartan, Université Henri Poincaré Nancy 1, B.P. 239, 54506 Vandoeuvre-les-Nancy Cedex, France (henrot@iecn.u-nancy.fr).

$\ddagger$ Antenne de Bretagne de l’ENS Cachan et IRMAR, Campus de Ker Lann, 35170-Bruz, France (pierre@bretagne.ens-cachan.fr).

§Université Hassan II - Mohammédia, Faculté des Sciences Ben M'Sik, Bp 7955 Casablanca, Maroc; and Antenne de Bretagne de l'ENS Cachan et IRMAR, Campus de Ker Lann, 35170-Bruz, France (rihani@bretagne.ens-cachan.fr).
} 
electromagnetic potential and is the solution of the Dirichlet problem

$$
-\Delta u_{\Omega}=f \text { in } \Omega, u_{\Omega}=0 \text { on } \partial \Omega,\left|\nabla u_{\Omega}\right| \rightarrow 0 \text { at } \infty
$$

Note that

$$
J(\Omega)=-\frac{1}{2} \int_{\Omega}\left|\nabla u_{\Omega}\right|^{2}=-\frac{1}{2} \int_{\Omega} f u_{\Omega} .
$$

The total energy of the system is the functional

$$
\Omega \rightarrow-\frac{1}{2} \int_{\Omega}\left|\nabla u_{\Omega}\right|^{2}+\tau P(\Omega),
$$

where $\tau>0$ and $P(\Omega)$ denotes the perimeter of $\Omega$ (or equivalently the perimeter of ${ }^{c} \Omega$ ). Here the second term $\tau P(\Omega)$ represents the surface tension energy.

We are interested in exterior shapes $\Omega^{*}$ for which the total energy reaches a minimum under the volume constraint

$$
S\left({ }^{c} \Omega\right)=S\left({ }^{c} \Omega^{*}\right)=S_{0},
$$

where $S(\cdot)$ denotes the Lebesgue measure in $\mathbb{R}^{2}$ and $S_{0}$ is prescribed. A necessary condition is that $\Omega^{*}$ be a critical shape for the functional

$$
\Omega \rightarrow E(\Omega)=-\frac{1}{2} \int_{\Omega}\left|\nabla u_{\Omega}\right|^{2}+\tau P(\Omega)-\Lambda S\left({ }^{c} \Omega\right),
$$

where $\Lambda$ is a Lagrange multiplier. This writes (see Section 2)

$$
\frac{1}{2}\left|\nabla u_{\Omega^{*}}\right|^{2}+\tau \mathcal{C}-\Lambda=0 \text { on } \partial \Omega^{*}
$$

where $\mathcal{C}$ is the curvature of $\partial \Omega^{*}$ seen from the exterior of $\Omega^{*}$ (so that it be positive if $\partial \Omega^{*}$ is a circle).

To decide whether such an equilibrium shape $\Omega^{*}$ is a minimum, we need to look at the positivity of the second derivative of the functional $E(\cdot)$ on an adequate functional subspace "tangent to the constraint". The study of this positivity is the main purpose of this paper.

A similar study has already been done in [17], but for the so-called "interior shaping" where the variable shape is a regular bounded open set. The expression of the second derivative is a very similar quadratic form except for a few signs, but which makes the question of positivity quite different. For instance, it is proved in [17] that any convex $\Gamma^{*}$ is stable. Here, we will consider only small analytic perturbations of a circle (therefore convex) and show that some are stable and some are not. The point is that we provide a complete numerical criterion to decide whether or not stability holds: it simply reduces to the elementary study of the positivity of a quadratic form in $\mathbb{R}^{2}$.

Note that a first analysis had been made in [9],[8],[16] in the particular case of zero surface tension, where instability mainly occurs for the exterior problem. We prove here that instability still occurs for small surface tension, but, more interesting, may even occur for large surface tension. We also refer to [1],[10],[11] for the study of second order conditions in shape optimization.

As a last comment, we refer to [5],[4] where the relationship between positivity and "true" stability of the equilibrium is discussed. Since we are in infinite dimension, 
strict positivity means coercivity with respect to a certain norm. But, as usually in shape optimization, the coercivity of the second derivative does not occur for the stronger norm where Taylor formula applies. Therefore, a local minimum may a priori not exist, even in a neighborhood for the strong norm. However, it is proved in [5],[4] that a local minimum does indeed exist, for this kind of functionals, at least for the stronger norm, that is to say, for regular perturbations of the equilibrium. On the other hand, examples are provided in [17] showing that "irregular" perturbations may decrease the energy while coercivity occurs.

2. Second derivative of exterior shaping functional. We consider the functional $E(\cdot)$ given by (1.2) where we assume that $f$ is regular (say $C^{1}$ ) and compactly supported. Let $\Omega^{*}$ be a regular open subset (say of class $C^{5}$ at least) such that ${ }^{c} \Omega^{*}$ is compact. As proved in several places (see e.g. [8],[9],[23],[20]), the functional $E(\cdot)$ is twice "differentiable" at $\Omega^{*}$. For the definition of these derivatives, we will adopt the point of view of [21] where Fréchet derivatives are considered in the space $\Theta_{3}$ of functions $\theta \in C^{3}\left(\mathbb{R}^{2}, \mathbb{R}^{2}\right)$ whose derivatives up to the third order are bounded. This space is equipped with a natural norm. Then the mapping

$$
\mathcal{E}: \theta \in \Theta_{3} \rightarrow E((I+\theta)(\Omega))
$$

is twice differentiable at $\theta=0$ (here $I$ denotes the identity in $\Theta_{3}$ ).

It is important to recall the structure of these derivatives. It is well-known for first derivatives from the pioneering paper by Hadamard [13]. It may also be found for the second derivative under different forms in several papers (see [20],[22], [7], [2], [23], [21]). Here, we use the notations and results of [21], Theorem 2.1 and Corollaries 2.4,2.9. We state it for our functional $E(\cdot)$, but it is the same for any regular functional. We denote by $\nu$ the inward normal unit vector to $\Gamma^{*}=\partial \Omega^{*}$ (directed towards $\Omega^{*}$ ). The Fréchet-derivatives of $\mathcal{E}(\cdot)$ at $\theta=0$ will be described by their action on arbitrary regular displacements $\xi, \eta \in \Theta_{3}$.

LEMMA 2.1. There exists a continuous linear map $l_{1}$ from $C^{3}\left(\Gamma^{*}\right)$ into $\mathbb{R}$ such that, for all $\xi \in \Theta_{3}$,

$$
\mathcal{E}^{\prime}(0)(\xi)=l_{1}(\xi \cdot \nu)
$$

There exists a continuous bilinear symmetric map $l_{2}$ from $C^{3}\left(\Gamma^{*}\right) \times C^{3}\left(\Gamma^{*}\right)$ into $\mathbb{R}$ such that for all $\xi, \eta \in \Theta_{3}$,

$$
\mathcal{E}^{\prime \prime}(0)(\xi, \eta)=l_{2}(\xi \cdot \nu, \eta \cdot \nu)-l_{1}\left(\xi^{\tau} \cdot D^{\tau} \nu \eta^{\tau}+\nu \cdot D^{\tau} \xi \eta^{\tau}+\nu \cdot D^{\tau} \eta \xi^{\tau}\right),
$$

where $\xi^{\tau}=\xi-(\xi \cdot \nu) \nu$ denotes the tangential component of $\xi$ and where $D^{\tau} \xi=D\left(\xi^{\tau}\right)$ denotes the matrix of the derivatives of $\xi^{\tau}$.

As a consequence of this structure theorem, to obtain full information on derivatives, it is sufficient to identify $l_{1}, l_{2}$. This may be done by using specific variations of the form $t \rightarrow E\left(\Omega_{t}\right)$ where $t$ tends to 0 and where the $\Omega_{t}$ 's are well-chosen perturbations of $\Omega^{*}$ (chosen to allow as simple computations as possible). To simplify the computation of the second derivative, it is interesting to choose normal displacements to $\Gamma^{*}$, so that only $l_{2}$ is involved.

Let us choose $\Omega_{t}=T_{t}\left(\Omega^{*}\right)$ where $T_{t}: \mathbb{R}^{2} \rightarrow \mathbb{R}^{2}$ is defined as follows. For any regular function $h: \Gamma^{*} \rightarrow \mathbb{R}$, we denote by $\widetilde{h}$ a regular extension of $h$ to $\mathbb{R}^{2}$ and by $\widetilde{\nu}$ a regular unitary extension of $\nu$ to $\mathbb{R}^{2}$ (see e.g. [12] for details). Now set

$$
\left.\begin{array}{c}
\forall x \in \mathbb{R}^{2}, \quad \forall t \geq 0: \quad T_{t} x=x+t \widetilde{h}(x) \widetilde{\nu}(x), \\
\Omega_{t}=T_{t}(\Omega), \quad e(t)=E\left(T_{t}(\Omega)\right)=E\left(\Omega_{t}\right) .
\end{array}\right\}
$$


Then, since $e(t)=\mathcal{E}(t \widetilde{h} \widetilde{\nu})$, by the chain rule and Lemma 2.1, we obtain

$$
e^{\prime}(0)=l_{1}(h), \quad e^{\prime \prime}(0)=l_{2}(h, h) .
$$

Now, we can explicitly describe $e^{\prime}(0), e^{\prime \prime}(0)$ in our case. We use the following notations:

$$
u=u_{\Omega^{*}}, \beta=\nabla u \cdot \nu=\partial u / \partial \nu\left(\text { interior normal derivative to } \Gamma^{*}\right) .
$$

We denote by $h^{\prime}$ the derivative with respect to the length parameter on $\Gamma^{*}$ and by $\mathcal{D}$ the exterior capacity operator on $\Gamma^{*}$ which, to each regular function $h: \Gamma^{*} \rightarrow \mathbb{R}$, associates the trace on $\Gamma^{*}$ of $-\partial H / \partial \nu=-(\nabla H)_{\Gamma^{*}} \cdot \nu$ where

$$
\Delta H=0 \text { in } \Omega^{*}, H=h \text { on } \Gamma^{*} \text { and }|\nabla H(x)| \rightarrow 0 \text { as }|x| \rightarrow \infty .
$$

(This operator is also called in the literature Dirichlet-to-Neumann or StekloffPoincaré operator. We refer for instance to [6] for its properties). In particular, we have

$$
\int_{\Gamma^{*}} h \mathcal{D} h=\int_{\Omega^{*}}|\nabla H|^{2}
$$

Recall that $\mathcal{C}$ denotes the curvature of $\Gamma^{*}$ seen from outside $\Omega^{*}$.

Proposition 2.2. With the above notations, the derivatives of $E(\cdot)$ are given as follows.

$$
e^{\prime}(0)=l_{1}(h)=\int_{\Gamma^{*}}\left(\frac{1}{2} \beta^{2}+\tau \mathcal{C}-\Lambda\right) h
$$

If $l_{1} \equiv 0$, then

$$
e^{\prime \prime}(0)=l_{2}(h, h)=\int_{\Gamma^{*}} \beta h \mathcal{D}(\beta h)-(2 \Lambda-\tau \mathcal{C}) \mathcal{C} h^{2}-f \beta h^{2}+\tau h^{\prime 2} .
$$

REMARK 2.3. As explained in the introduction, " $l_{1} \equiv 0 "$ means that $\Omega^{*}$ is a critical shape for $\Omega \rightarrow J(\Omega)+\tau P(\Omega)$ (see 1.2) under the constraint $S\left({ }^{c} \Omega\right)=S_{0}$, and $\Lambda$ is the Lagrange multiplier. In this case, we have

$$
\beta^{2}=2(\Lambda-\tau \mathcal{C}) \text { on } \Gamma^{*}
$$

REMARK 2.4. Indications for the proof of Proposition 2.2 may be found in the appendix. The computation is very similar to the one made for the so-called "interior shaping problem" (see [17]). The only difference here is that $\Omega^{*}$ is an exterior domain whose complement has a prescribed measure, while in [17], $\Omega^{*}$ is a bounded domain with prescribed measure. This leads to slight differences in the expression of the derivatives (but to important differences for the question of positivity).

REMARK 2.5. A necessary second order condition: Let us assume that $J(\cdot)+\tau P(\cdot)$ has a local minimum at $\Omega^{*}$ among the regular domains $\Omega$ satisfying the measure constraint $S\left({ }^{c} \Omega\right)=S_{0}$. Then $\theta \in \Theta_{3} \rightarrow(J+\tau P)\left((I+\theta)\left(\Omega^{*}\right)\right)$ has a 
minimum at $\theta=0$ on the manifold of $\theta$ 's such that $S\left((I+\theta)\left({ }^{c} \Omega^{*}\right)\right)=S\left({ }^{c} \Omega^{*}\right)$. As a consequence, the second derivative of the full Lagrangian

$$
\theta \rightarrow(J+\tau P-\Lambda S)\left((I+\theta)\left(\Omega^{*}\right)\right)
$$

is nonnegative on the hyperplane of linearized constraints, that is on $\left\{\xi \in \Theta_{3} ; \int_{\Gamma^{*}} \xi\right.$. $\nu=0\}$. This means that, for all $h \in C^{3}\left(\Gamma^{*}\right)$ such that $\int_{\Gamma^{*}} h=0$, the quadratic form

$$
Q(h)=\int_{\Gamma^{*}} \beta h \mathcal{D}(\beta h)-(2 \Lambda-\tau \mathcal{C}) \mathcal{C} h^{2}-f \beta h^{2}+\tau h^{\prime 2},
$$

is nonnegative.

REMARK 2.6. From now on, we will mainly assume that $f$ vanishes on $\Gamma^{*}$. As explained in [14],[17],[3], it is actually natural to assume that $f$ is even compactly supported in $\Omega^{*}$. Moreover, once we have studied the case $f=0$ on $\Gamma^{*}$, we may separately study the influence of adding the term in $f$ in the expression of $l_{2}(h, h)$ above. A simple analysis shows that it essentially brings more instability. If for instance $f \geq 0$, then $u \geq 0$ on $\Omega^{*}$ and $\beta=\partial u / \partial \nu \geq 0$ on $\Gamma^{*}$. Therefore, the extra term $-f \beta h^{2}$ in $Q(h)$ is negative.

Note that, if $f=0$ on $\Gamma^{*}$, the quadratic form $Q$ depends only on the geometry of $\Gamma^{*}$ and on $\tau, \Lambda$.

3. Positivity of second derivatives. Following the above discussion, our goal is to study, in some particular situations, the positivity of the quadratic form

$$
h \rightarrow Q(h)=\int_{\Gamma^{*}} \beta h \mathcal{D}(\beta h)-(2 \Lambda-\tau \mathcal{C}) \mathcal{C} h^{2}+\tau h^{\prime 2}
$$

for functions $h$ satisfying $\int_{\Gamma^{*}} h=0$. Obviously, $Q$ is continuous for the $H^{1}\left(\Gamma^{*}\right)$ topology so that the natural question is to decide whether $Q$ is coercive on the space

$$
Z_{0}^{1}\left(\Gamma^{*}\right)=\left\{h \in H^{1}\left(\Gamma^{*}\right): \int_{\Gamma^{*}} h=0\right\}
$$

that is whether

$$
\exists \eta_{0}>0, \text { such that } \forall h \in Z_{0}^{1}\left(\Gamma^{*}\right), \quad Q(h) \geq \eta_{0}\|h\|_{H^{1}\left(\Gamma^{*}\right)}^{2} .
$$

We will restrict our study to the case where $\Gamma^{*}$ is a small analytic perturbation of the unit circle $\Gamma_{0}$ of the form

$$
\left.\Gamma^{*}=\left\{\phi_{\epsilon}\left(e^{i \theta}\right), \theta \in[0,2 \pi]\right\}, \quad \phi_{\epsilon}(z)=z+\epsilon R(z)+\epsilon^{2} T_{\epsilon}(z)\right\}
$$

with $\epsilon>0$ small, $R, T_{\epsilon}$ are complex valued holomorphic functions in a neighborhood of the unit circle and $T_{\epsilon}$ is uniformly bounded as well as its derivatives in a neighborhood of the unit circle. In particular, $\Gamma^{*}$ is a Jordan curve.

Two families of situations: It turns out that there are two rather different families of situations: indeed, the equilibrium condition (2.7) writes

$$
\beta^{2}=2(\Lambda-\tau \mathcal{C}) \text { on } \Gamma^{*}
$$


so that, if $\mathcal{C}_{M}:=\max _{\Gamma^{*}} \mathcal{C}$, then $\Lambda \geq \tau \mathcal{C}_{M}$.

But the situation will be different depending on whether this inequality is strict or not.

If there exists a point of $\Gamma^{*}$ at which $\nabla u=0$, then necessarily $\tau \mathcal{C}_{M}=\Lambda$. This is necessarily the case if, for instance, $\int_{\Omega^{*}} f=0$ and $\Gamma^{*}$ is a Jordan curve. Indeed, if $\nabla u$ did not vanish on $\Gamma^{*}$, then, we would have

$$
\int_{\Gamma^{*}}|\nabla u|=\left|\int_{\Gamma^{*}} \nabla u \cdot \nu\right|=\left|\int_{\Omega^{*}} \Delta u\right|=\left|\int_{\Omega^{*}} f\right|=0,
$$

which would be a contradiction with $\nabla u \neq 0$ on $\Gamma^{*}$. In some applications, like the shaping of liquid metals by electromagnetic devices, this case $\tau \mathcal{C}_{m}=\Lambda$ turns out to be more "physical" since, in two-dimensional models, it is natural to assume that the distribution of currents $f$ satisfies $\int_{\Omega^{*}} f=0$ (see [9],[8],[14]).

We will see that small perturbations of the disk may be stable or not and this will depend only on three quantities $a, b, c$ that we introduce now. Assuming $\Re(\cdot)$ denotes the real part of a complex number, set

$$
\begin{array}{r}
g_{1}(\theta)=\Re\left(\left(R^{\prime}\left(e^{i \theta}\right)\right), g_{2}(\theta)=\Re\left(e^{i \theta} R^{\prime \prime}\left(e^{i \theta}\right)-R^{\prime}\left(e^{i \theta}\right)\right),\right. \\
g_{2}^{\infty}=\sup \left\{g_{2}(\theta), \theta \in[0,2 \pi]\right\}, \eta=\operatorname{sign}(\beta), \ell(\theta)=\eta \sqrt{g_{2}^{\infty}-g_{2}(\theta)} .
\end{array}
$$

Next, we set $\left(\mathcal{D}^{0}\right.$ denotes the exterior capacity operator $\mathcal{D}$ on the unit circle $\left.\Gamma_{0}\right)$ :

$$
\left\{\begin{array}{l}
a=\int_{0}^{2 \pi}\left[2 \ell(\theta) \cos \theta \mathcal{D}^{0}(\ell(\theta) \cos \theta)-g_{2}^{\infty}-g_{1}(\theta)\right] d \theta, \\
b=\int_{0}^{2 \pi}\left[2 \ell(\theta) \sin \theta \mathcal{D}^{0}(\ell(\theta) \sin \theta)-g_{2}^{\infty}-g_{1}(\theta)\right] d \theta \\
c=\int_{0}^{2 \pi}\left[2 \ell(\theta) \cos \theta \mathcal{D}^{0}(\ell(\theta) \sin \theta)\right] d \theta
\end{array}\right.
$$

Theorem 3.1. Assume $\Lambda=\tau \mathcal{C}_{M}$. Then, if

$$
a>0, b>0, c^{2}-a b<0,
$$

there exists $\epsilon_{0}>0$ such that for all $\left.\epsilon \in\right] 0, \epsilon_{0}\left[, Q\right.$ satifies the $H^{1}$-coercivity property (3.2). On the other hand, if

$$
a<0 \text { or } b<0 \text { or } c^{2}-a b>0,
$$

then, for $\epsilon$ small enough, there exists $h \in Z_{0}^{1}\left(\Gamma^{*}\right)$ such that $Q(h)<0$.

REMARK 3.2. Above theorem says that the positivity or the nonpositivity of $Q$ depends only of the positivity of the 2-dimensional quadratic form

$$
(x, y) \in \mathbb{R}^{2} \rightarrow a x^{2}+2 c x y+b y^{2} .
$$

Therefore, the positivity of $Q$ may be decided from quite elementary computations.

As one easily checks, both (3.8) and (3.9) can happen. As a consequence, some small analytic perturbations of circles are stable, somes others are unstable. The next corollary describes a "good" situation: 
Corollary 3.3. Assume $R(z)=z^{-(2 k+1)}, k \geq 1$. Then, for $\epsilon$ small enough, the coercivity property (3.2) holds.

REMARK 3.4. As proved in [14], any analytic Jordan curve $\Gamma^{*}$ for which the curvature reaches its maximum at an even number of points is "shapable" with $\tau \mathcal{C}_{M}=$ $\Lambda$, in the sense that there exists a distribution $f$ compactly supported in $\Omega^{*}$ whose equilibrium shape is exactly $\Gamma^{*}$ and for which $\tau \mathcal{C}_{M}=\Lambda$. Obviously, the curves considered in the above corollary have this geometric property (so that it makes sense to consider them in the scope of Theorem 3.1).

Corollary 3.5. Assume $\Gamma^{*}$ is an equilibrium curve corresponding to a distribution $f$ of four Dirac masses at the vertices $(\alpha, \beta),(-\alpha, \beta),(-\alpha,-\beta),(\alpha,-\beta)$ of a rectangle and with alternate sign. Assume $\tau$ is large. Then, there exist values of $(\alpha, \beta)$ (with $|\alpha| \neq|\beta|$ ) for which instability holds.

REMARK 3.6. It may be surprising to consider the case where $f$ is a sum of Dirac masses since we have assumed that $f$ is at least a $C^{1}$ function. Indeed, due the presence of Dirac masses, the Dirichlet energy is even infinite! However, $f$ is zero around $\Gamma^{*}$ and the corresponding quadratic form does exist and is well defined on a curve which is close to a circle for $\tau$ large. Therefore, it makes sense to analyze the positivity of this form. Now, a little work is needed to interpret the result and to redefine the notion of minimum and stability after extracting the singularities. We will not provide the details here, but just look at the positivity of $Q$. We refer to [15] for more information on the case of Dirac masses. Note that it is also significant of the situation where $f$ is regular and close to a sum of Dirac masses.

Finally, we consider the case $\Lambda>\tau \mathcal{C}_{M}$. As one expects, we obtain quite less stability in that situation. For instance, we have

ThEOREM 3.7. Assume $\Omega^{*}$ is the exterior of a regular Jordan curve $\Gamma^{*}$ different from a circle and $\Lambda>\tau \mathcal{C}_{M}$. Then, there exist $h \in Z_{0}^{1}\left(\Gamma^{*}\right)$ and $\tau_{0}>0$ such that $Q(h)<0$ for all $\tau \in\left(0, \tau_{0}\right)$.

REMARK 3.8. This is an easy consequence of the instability result proved in [9] for $\tau=0$. It is interesting to notice that instability is not only due to the absence of surface tension but remains for small surface tension.

REMARK 3.9. As in Remark 3.4, to understand the above theorem, we must recall that (see [14]), given any analytic Jordan curve $\Gamma^{*}$ and given any $\tau, \Lambda$ with $\Lambda>\tau \mathcal{C}_{M}$, there exists a distribution $f$ compactly supported in $\Omega^{*}$ whose equilibrium shape is $\Gamma^{*}$. The theorem says that, for this set of data, instability occurs since the corresponding second derivative is negative in some direction.

Let us now consider the case where $\Gamma^{*}$ is a perturbation of the unit circle as in (3.3). Like for Theorem 3.1, we introduce convenient quantities:

$$
\left\{\begin{array}{l}
\widehat{a}=\int_{0}^{2 \pi}\left[-\Lambda\left(g_{1}+g_{2}\right)+\tau g_{1}\right] \cos 2 \theta, \\
\widehat{c}=\int_{0}^{2 \pi}\left[-\Lambda\left(g_{1}+g_{2}\right)+\tau g_{1}\right] \sin 2 \theta .
\end{array}\right.
$$


THEOREM 3.10. Assume $\Gamma^{*}$ is a perturbation of the unit circle as in (3.3) and $\Lambda>\tau \mathcal{C}_{M}$. If

$$
\widehat{a} \neq 0 \quad \text { or } \quad \widehat{c} \neq 0
$$

then, for $\epsilon$ small enough, there exists $h \in Z_{0}^{1}\left(\Gamma^{*}\right)$ such that $Q(h)<0$.

REMARK 3.11. This theorem says that, "in general", small perturbations of circle are unstable when $\Lambda>\tau \mathcal{C}_{m}$. When $\widehat{a}=\widehat{c}=0$, it is necessary to pursue further the expansion with respect to the small parameter $\epsilon$ to decide about stability. But, since the dependence of $\widehat{a}, \widehat{c}$ in $g_{1}, g_{2}$ is linear, this happens only for a subspace of codimension 2. It turns out that it is precisely the case for the perturbations of the kind considered in Corollary 3.3, except for $k=1$ (the case of the ellipse). Actually, we have:

Corollary 3.12. Assume $\Lambda>\tau \mathcal{C}_{M}$ and the perturbation is of the form $R(z)=$ $\sum_{n \geq 1} \sigma_{n} z^{-n}$ with $\sigma_{n} \in \mathbb{R}$. Then, if $\sigma_{1} \neq 0$, instability holds for $\epsilon$ small enough.

REMARK 3.13. The proof of the results are given in Section 5. They requires some abstract tools of independent interest about the positivity of the restriction of quadratic forms on hyperplanes in real Hilbert spaces. They are discussed in the next section.

4. A general criterion for constrained stability. Let $\mathcal{H}$ be a real separable Hilbert space with scalar product and norm denoted by $(\cdot, \cdot),\|\cdot\|$, let $\mathcal{H}^{\prime}$ its dual with $\mathcal{H}^{\prime} \times \mathcal{H}$ duality product $\left\langle\cdot, \cdot>\right.$ and $\mathcal{R}$ the Riesz isomorphism from $\mathcal{H}$ onto $\mathcal{H}^{\prime}$. Let $\phi: \mathcal{H} \times \mathcal{H} \rightarrow \mathbb{R}$ be a symmetric continuous bilinear form on $\mathcal{H}, Q$ the associated quadratic form $Q(h)=\phi(h, h)$ for all $h \in \mathcal{H}$ and $A$ the linear continuous symmetric operator from $\mathcal{H}$ into $\mathcal{H}^{\prime}$ such that $\forall h \in \mathcal{H},<A h, h>=Q(h)$.

We are interested in the positivity of the restriction of $Q$ to hyperplanes of $\mathcal{H}$. Note that, if $Q$ writes in a Hilbert basis $\left(e_{i}\right)_{i \geq 1}$

$$
Q(h)=\sum_{i \geq 1} \lambda_{i} h_{i}^{2}, \text { for } h=\sum_{i \geq 1} h_{i} e_{i},
$$

then the above question has a trivial answer in the two following situations: if $\forall i \geq$ $1, \lambda_{i}>0$, then $Q$ is positive everywhere; if $\lambda_{1} \leq \lambda_{2}<0$, the restriction of $Q$ to any hyperplane has at least one direction in which it is negative. Thus, the interesting case is when only one eigenvalue is negative. For this, we define:

Definition 4.1. We will say that $Q$ is of type $(-1,+\infty)$ if the two following conditions hold:

There exists $h \in \mathcal{H}$ such that $Q(h)<0$.

One cannot find linearly independent $h_{1}, h_{2} \in \mathcal{H}$ such that

$\phi\left(h_{1}, h_{2}\right)=0 \quad, \quad Q\left(h_{1}\right) \leq 0 \quad, \quad Q\left(h_{2}\right) \leq 0$.

REMARK 4.2. If $Q$ is given by (4.1), it is easy to see that it is of type $(-1,+\infty)$ if and only if, up to a reordering

$$
\lambda_{1}<0, \quad \forall i \geq 2, \lambda_{i}>0 .
$$


More generally, we have the following lemma.

LEMma 4.3. The quadratic form $Q$ is of type $(-1,+\infty)$ if and only if there exists a closed subspace $\mathcal{V}$ of $\mathcal{H}$ and $h_{0} \in \mathcal{H} \backslash\{0\}$ such that

$$
\mathcal{H}=\mathcal{V} \oplus\left\{h_{0}\right\}, \quad Q\left(h_{0}\right)<0,
$$

and the restriction of $Q$ to $\mathcal{V} \backslash\{0\}$ is positive. If, moreover,

$$
\exists \alpha>0 \quad, \forall v \in \mathcal{V}, \quad Q(v) \geq \alpha\|v\|^{2},
$$

then $A$ is invertible.

We now come to the main statement of this section.

Theorem 4.4. Assume $Q$ is of type $(-1,+\infty)$ and $A$ is invertible. Let $w \in \mathcal{H} \backslash\{0\}$ and let $w^{\perp}$ be the hyperplane orthogonal to $w$. Then

$$
\text { The restriction of } Q \text { to } w^{\perp} \backslash\{0\} \text { is positive, }
$$

if and only if

$$
Q\left(A^{-1} \circ \mathcal{R}(w)\right)<0
$$

If moreover, (4.5) is satisfied on some hyperplane $\mathcal{V}$, then so it is on $w^{\perp}$.

REMARK 4.5. The interest of the above theorem is that it provides a numerical criterion for constrained stability. This will turn out to be quite useful when looking at quadratic forms depending on a (small) parameter. We refer to [18],[19] for a systematic study of other criteria and for the case of more constraints.

We will strongly use this criterion to prove the results of Section 3, but, just to make it more explicit, let us describe now two independent illustrations.

Corollary 4.6. Assume that $Q$ is given as in (4.1) and satisfies (4.4) and that $A$ is invertible. Then, its restriction to $w^{\perp} \backslash\{0\}$, where $w=\sum_{i \geq 1} w_{i} e_{i}$ is positive if and only if

$$
\sum_{i \geq 1} \frac{w_{i}^{2}}{\lambda_{i}}<0
$$

COROLlary 4.7. Let $\mathcal{H}=H^{1 / 2}(\Gamma)$ where $\Gamma$ is a regular Jordan curve with exterior $\Omega$. Assume the following quadratic form on $\mathcal{H}$ is of type $(-1,+\infty)$

$$
\forall h \in \mathcal{H}, Q(h)=\int_{\Gamma} h \mathcal{D} h-\sigma h^{2},
$$

where $\sigma: \Gamma \rightarrow \mathbb{R}$ is regular. Assume $A$ is invertible. Let $z \in \mathcal{H} \backslash\{0\}$ and $\mathcal{H}_{z}=\{h \in$ $\left.\mathcal{H} ; \int_{\Gamma} z h=0\right\}$. Then, the restriction of $Q$ to $\mathcal{H}_{z} \backslash\{0\}$ is positive if and only if

$$
\int_{\Gamma} u_{0} z<0
$$


where $u_{0}$ is the solution of

$$
\Delta u_{0}=0 \text { in } \Omega, \quad \nabla u_{0} \rightarrow 0 \text { at } \infty, \quad-\frac{\partial u_{0}}{\partial \nu}-\sigma u_{0}=z \text { on } \Gamma .
$$

Remark 4.8. Conditions of type (4.8) have been used in [9],[16] to study the positivity of this $Q$ on the space $\left\{h \in H^{1 / 2}(\Gamma) ; \int_{\Gamma} h=0\right\}$. This form $Q$ appears for our problem in the limit case $\tau=0$. For instance, as proved in [9], there is always a direction of instability if $\sigma$ is the curvature of $\Gamma$.

Proof of Lemma 4.3. Assume $Q$ is of type $(-1,+\infty)$. By (4.2), there exists $h_{0} \in \mathcal{H}$ with $Q\left(h_{0}\right)<0$. Now let $\mathcal{V}:=\left\{v \in \mathcal{H} ; \phi\left(h_{0}, v\right)=0\right\}$. Since $h_{0} \notin \mathcal{V}, \mathcal{H}=\mathcal{V} \oplus\left\{h_{0}\right\}$. Moreover, the restriction of $Q$ to $\mathcal{V} \backslash\{0\}$ is strictly positive. Indeed, if it was not the case, there would be $v \in \mathcal{V} \backslash\{0\}$ such that $Q(v) \leq 0$ and this would contredict the assumption (4.3).

Assume now that we have $h_{0}, \mathcal{V}$ as above. Then (4.2) holds. Assume, by contradiction, that there exist linearly independent $h_{1}, h_{2}$ such that

$$
\phi\left(h_{1}, h_{2}\right)=0, Q\left(h_{1}\right) \leq 0, Q\left(h_{2}\right) \leq 0 .
$$

Then, the restriction of $Q$ to the subspace spanned by $h_{1}, h_{2}$ is nonpositive. But this 2-dimensional subspace necessarily intersects $\mathcal{V} \backslash\{0\}$ where $Q$ is positive. This is a contradiction.

For the invertibility of $A$, if $h=v+\mu h_{0}, v \in \mathcal{V}$ and $\mu \in \mathbb{R}$, we have

$$
<A h, v-\mu h_{0}>=Q(v)-\mu^{2} Q\left(h_{0}\right) \geq \beta\left(\|v\|^{2}+\mu^{2}\right),
$$

where $\beta=\min \left\{\alpha,-Q\left(h_{0}\right)\right\}$. But we also have, for some $\gamma>0$,

$$
\|v\|^{2}+\mu^{2} \geq \gamma\left\|v+\mu h_{0}\right\|\left\|v-\mu h_{0}\right\| .
$$

It follows from these inequalities, after simplifying by $\left\|v-\mu h_{0}\right\|$, that

$$
\forall v \in \mathcal{V}, \forall \mu \in \mathbb{R},\left\|A\left(v+\mu h_{0}\right)\right\| \geq \beta \gamma\left\|v+\mu h_{0}\right\| .
$$

This proves the invertibility of $A$.

Proof of Theorem 4.4. Assume (4.7). For $w_{1}=A^{-1} \circ \mathcal{R}(w)$, we have $Q\left(w_{1}\right)<0$. Assume by contradiction that (4.6) does not hold, which means that there exists $w_{2} \in w^{\perp} \backslash\{0\}$ such that $Q\left(w_{2}\right) \leq 0$. Then

$$
0=\left(w, w_{2}\right)=\left(\mathcal{R}^{-1} \circ A\left(w_{1}\right), w_{2}\right)=<A w_{1}, w_{2}>=\phi\left(w_{1}, w_{2}\right) .
$$

Moroever, $w_{1}, w_{2}$ are linearly independent, because if we had $w_{2}=\theta w_{1}, \theta \neq 0$, then we would have $0=\phi\left(w_{1}, \theta w_{1}\right)=\theta Q\left(w_{1}\right)$ which is not the case. The existence of such $w_{1}, w_{2}$ contradicts the fact that $Q$ is of type $(-1,+\infty)$.

In the opposite direction, suppose now (4.6). Assume by contradiction that $Q \circ$ $A^{-1} \circ \mathcal{R}(w) \geq 0$. With the same notations as above, this writes $Q\left(w_{1}\right) \geq 0$ and even $Q\left(w_{1}\right)>0$ : indeed, since $Q\left(w_{1}\right)=\phi\left(w_{1}, w_{1}\right)=\left(w, w_{1}\right), " Q\left(w_{1}\right)=0$ " would imply $w_{1} \in w^{\perp} \backslash\{0\}$, but $Q$ is assumed to be positive (strictly) on $w^{\perp} \backslash\{0\}$. We now introduce

$$
\mathcal{H}_{2}=\left\{h_{2} \in \mathcal{H} ; 0=\phi\left(w_{1}, h_{2}\right)=<A w_{1}, h_{2}>=\left(\mathcal{R}^{-1} \circ A\left(w_{1}\right), h_{2}\right)=\left(w, h_{2}\right)\right\},
$$


that is $\mathcal{H}_{2}=w^{\perp}$. We have $\mathcal{H}=\left\{w_{1}\right\} \oplus \mathcal{H}_{2}$ since $w_{1} \notin \mathcal{H}_{2}\left(<A w_{1}, w_{1}>=Q\left(w_{1}\right)>0\right)$. Now, for all $w_{2}=\lambda w_{1}+h_{2}, \lambda \in \mathbb{R}, h_{2} \in \mathcal{H}_{2}$, we have by (4.6)

$$
Q\left(w_{2}\right)=\lambda^{2} Q\left(w_{1}\right)+Q\left(h_{2}\right) \geq 0, \quad\left(Q\left(w_{1}\right)>0\right) .
$$

Thererefore $Q$ is nonnegative on the whole space $\mathcal{H}$ which contradicts the fact that it is of type $(-1,+\infty)$.

Assume that, moreover, $Q$ satisfies (4.5). We also have $\mathcal{H}=\mathcal{V} \oplus\left\{w_{1}\right\}$ and any $h_{2} \in w^{\perp} \backslash\{0\}$ may be written $h_{2}=v_{2}+\lambda w_{1}, v_{2} \in \mathcal{V}, \lambda \in \mathbb{R}$. Since $\phi\left(h_{2}, w_{1}\right)=$ $\left(h_{2}, w\right)=0$, we have $Q\left(v_{2}\right)=Q\left(h_{2}\right)+\lambda^{2} Q\left(w_{1}\right)$ so that, with $\gamma=\min \left\{\alpha,-Q\left(w_{1}\right)\right\}$

$$
Q\left(h_{2}\right) \geq \gamma\left(\lambda^{2}+\left\|v_{2}\right\|^{2}\right) \geq \gamma^{\prime}\left\|v_{2}+\lambda w_{1}\right\|^{2},
$$

for some $\gamma^{\prime}>0$. The last part of the theorem follows.

Proof of Corollary 4.6. Assumptions of Theorem 4.4 are satisfied. We just have to make the criterion explicit. Here $\mathcal{H}$ may be identified with $\ell^{2}(\mathbb{N})$ as well as its dual $\mathcal{H}^{\prime}$ and the Riesz isomorphism $\mathcal{R}$ is just the identity. The operator is the multiplication by $\lambda_{i}$ in each direction $e_{i}$; therefore $A^{-1}$ is just the mutiplication by $\lambda_{i}^{-1}$ in each direction $\lambda_{i}$. The new expression of the criterion follows.

Proof of Corollary 4.7. Let us choose on $\mathcal{H}=H^{1 / 2}(\Gamma)$ the norm defined by

$$
\|u\|^{2}=\int_{\Gamma} u^{2}+u \mathcal{D} u=\int_{\Gamma} u \cdot(I+\mathcal{D}) u .
$$

Then, the Riesz isomorphism from $\mathcal{H}$ into $\mathcal{H}^{\prime}=H^{-1 / 2}(\Gamma)$ is $I+\mathcal{D}$ and we have

$$
\forall h_{1}, h_{2} \in \mathcal{H}, \int_{\Gamma} h_{1} h_{2}=\left((I+\mathcal{D})^{-1} h_{1}, h_{2}\right)_{\mathcal{H} \times \mathcal{H}}=<h_{1}, h_{2}>_{\mathcal{H}^{\prime} \times \mathcal{H}} .
$$

Let $A=\mathcal{D}-\sigma I$ be the operator such that $Q(h)=<A h, h>_{\mathcal{H}^{\prime}} \times \mathcal{H}$. Let $z$ and $u_{0}$ be given as in the corollary. Note that $u_{0_{\mid \Gamma}}=A^{-1}(z)$ and

$$
H_{z}=\left\{h \in \mathcal{H} ; 0=\int_{\Gamma} z h=<z, h>_{\mathcal{H}^{\prime} \times \mathcal{H}}=\left(\mathcal{R}^{-1}(z), h\right)_{\mathcal{H} \times \mathcal{H}}\right\},
$$

so that $H_{z}=\left[\mathcal{R}^{-1}(z)\right]^{\perp}$ and the criterion (4.7) writes

$$
0>Q\left(A^{-1} z\right)=<z, A^{-1} z>_{\mathcal{H}^{\prime} \times \mathcal{H}}=\int_{\Gamma} z u_{0} .
$$

This ends the proof of the corollary.

5. The proofs of the results of Section 3. In this section, we will prove the results of Section 3. We start with the following technical lemma

Lemma 5.1. Let $\mathcal{V}$ be a real Hilbert space with norm $\|\cdot\|, \mathcal{V}_{1}$ and $\mathcal{V}_{2}$ closed subspaces of $\mathcal{V}$ such that $\mathcal{V}=\mathcal{V}_{1} \oplus \mathcal{V}_{2}$. Let $Q_{1}, Q_{2}$ be continuous quadratic forms on $\mathcal{V}$ and $\phi_{1}, \phi_{2}$ the associated bilinear forms. Assume

$$
\begin{aligned}
& \forall v_{1} \in \mathcal{V}_{1} \quad, \quad \forall v_{2} \in \mathcal{V}_{2}, \quad \phi_{1}\left(v_{1}, v_{2}\right)=0, \\
& \exists \alpha_{1}>0, \quad \forall v_{1} \in \mathcal{V}_{1}, \quad Q_{1}\left(v_{1}\right) \geq \alpha_{1}\left\|v_{1}\right\|^{2}, \\
& \forall v_{2} \in \mathcal{V}_{2}, \quad Q_{1}\left(v_{2}\right)=0, \\
& \exists \alpha_{2}>0, \quad \forall v_{2} \in \mathcal{V}_{2}, \quad Q_{2}\left(v_{2}\right) \geq \alpha_{2}\left\|v_{2}\right\|^{2} .
\end{aligned}
$$


Then, there exists $\epsilon_{0}>0$ and $\alpha>0$ such that, for all $\left.\epsilon \in\right] 0, \epsilon_{0}[$, the quadratic form $Q_{\epsilon}=Q_{1}+\epsilon Q_{2}$ satisfies

$$
\forall v \in \mathcal{V} \quad, \quad Q_{\epsilon}(v) \geq \epsilon \alpha\|v\|^{2} .
$$

Proof of Lemma 5.1. Let $v \in \mathcal{V}$. We write $v=v_{1}+v_{2}, v_{1} \in \mathcal{V}_{1}$ and $v_{2} \in \mathcal{V}_{2}$. We have, using (5.1) and (5.3)

$$
Q_{\epsilon}(v)=Q_{1}\left(v_{1}\right)+\epsilon\left[Q_{2}\left(v_{1}\right)+Q_{2}\left(v_{2}\right)+2 \phi_{2}\left(v_{1}, v_{2}\right)\right] .
$$

By (5.2) and (5.4)

$$
Q_{\epsilon}(v) \geq \alpha_{1}\left\|v_{1}\right\|^{2}+\epsilon\left(\alpha_{2}\left\|v_{2}\right\|^{2}-M_{2}\left\|v_{1}\right\|^{2}-2 M_{2}\left\|v_{1}\right\|\left\|v_{2}\right\|\right),
$$

where $M_{2}$ is the continuity constant of $\phi_{2}$. Using that for all $\eta>0$

$$
2\left\|v_{1}\right\|\left\|v_{2}\right\| \leq \frac{1}{\eta}\left\|v_{1}\right\|^{2}+\eta\left\|v_{2}\right\|^{2}
$$

we deduce

$$
Q_{\epsilon}(v) \geq \alpha_{1}\left\|v_{1}\right\|^{2}+\epsilon\left[\left(\alpha_{2}-M_{2} \eta\right)\left\|v_{2}\right\|^{2}-M_{2}\left(1+\frac{1}{\eta}\right)\left\|v_{1}\right\|^{2}\right] .
$$

We choose $\eta>0$ small enough so that $\alpha_{2}-M_{2} \eta \geq \alpha_{2} / 2$, and $\epsilon_{0}>0$ small enough so that $\alpha_{1}-\epsilon_{0} M_{2}\left(1+\frac{1}{\eta}\right) \geq \epsilon_{0} \alpha_{1} / 2$. Then, choosing $\gamma=\min \left\{\alpha_{1}, \alpha_{2}\right\} / 2$, we have for all $v \in V$ and all $\epsilon \in\left(0, \epsilon_{0}\right)$

$$
Q_{\epsilon}(v) \geq \epsilon \gamma\left(\left\|v_{1}\right\|^{2}+\left\|v_{2}\right\|^{2}\right) \geq \epsilon \alpha\left\|v_{1}+v_{2}\right\|^{2},
$$

for some $\alpha$ depending only on $\gamma$ and on the decomposition $\mathcal{V}=\mathcal{V}_{1} \oplus \mathcal{V}_{2}$. Then, (5.5) follows.

LEMMA 5.2. The quadratic form (3.1) can be rewritten on the unit circle $\Gamma_{0}$ as

$$
Q(h)=\mathcal{Q}(H)=\int_{\Gamma_{0}} \beta H \mathcal{D}^{0}(\beta H)-(2 \Lambda-\tau \mathcal{C}) \mathcal{C} H^{2}\left|\phi_{\epsilon}^{\prime}\right|+\tau \frac{H^{\prime 2}}{\left|\phi_{\epsilon}^{\prime}\right|},
$$

where $\beta^{2}=2\left[\Lambda-\tau \mathcal{C}\left(\phi_{\epsilon}\left(e^{i \theta}\right)\right)\right]$ and $H(\theta):=h\left(\phi_{\epsilon}\left(e^{i \theta}\right)\right), \theta \in[0,2 \pi]$. When $\Lambda=\tau \mathcal{C}_{M}$, this becomes $(\eta=\operatorname{sign}(\beta))$

$$
\mathcal{Q}(H)=\tau \int_{\Gamma_{0}} 2 \eta H \sqrt{\mathcal{C}_{M}-\mathcal{C}} \mathcal{D}^{0}\left(\eta H \sqrt{\mathcal{C}_{M}-\mathcal{C}}\right)-\left(2 \mathcal{C}_{M}-\mathcal{C}\right) \mathcal{C} H^{2}\left|\phi_{\epsilon}^{\prime}\right|+\frac{H^{\prime 2}}{\left|\phi_{\epsilon}^{\prime}\right|}
$$

Proof. We transfer the quadratic form (3.1) on the unit circle $\Gamma_{0}$ by setting as in (3.3), $\Gamma^{*}=\left\{\phi_{\epsilon}\left(e^{i \theta}\right), \theta \in[0,2 \pi]\right\}$. Note that, for all $g \in H^{1}\left(\Gamma^{*}\right)$ and $G(\theta)=g\left(\phi_{\epsilon}\left(e^{i \theta}\right)\right)$, one has

$$
(\mathcal{D} g)\left(\phi_{\epsilon}\left(e^{i \theta}\right)\right)\left|\phi_{\epsilon}^{\prime}\left(e^{i \theta}\right)\right|=\mathcal{D}^{0} G .
$$

By change of variable, this implies $\int_{\Gamma^{*}} g \mathcal{D} g=\int_{\Gamma_{0}} G \mathcal{D}^{0} G$. For the last term in (5.6), we recall that the length parameter on $\Gamma^{*}$ is given by $s=\int_{0}^{\theta}\left|\phi_{\epsilon}^{\prime}\left(e^{i \varphi}\right)\right| d \varphi$ and we make the appropriate change of variable. 
LEMMA 5.3. Assume $\Lambda=\tau \mathcal{C}_{M}$, then the quadratic form $\mathcal{Q}$ can be written

$$
\mathcal{Q}=\tau\left[\mathcal{Q}_{1}+\epsilon \mathcal{Q}_{2}+r_{\epsilon}\right]
$$

where

$$
\begin{aligned}
\mathcal{Q}_{1}(H) & =\int_{\Gamma_{0}} H^{\prime 2}-H^{2}=-\int_{\Gamma_{0}} H\left(H^{\prime \prime}+H\right), \\
\mathcal{Q}_{2}(H) & =\int_{\Gamma_{0}} 2 \ell H \mathcal{D}^{0}(\ell H)-\left(2 g_{2}^{\infty}+g_{1}\right) H^{2}-g_{1} H^{\prime 2},\left(\ell=\eta \sqrt{g_{2}^{\infty}-g_{2}}\right), \\
\left|r_{\epsilon}(H)\right| & \leq c \epsilon^{2}\|H\|_{H^{1}\left(\Gamma_{0}\right)}^{2},
\end{aligned}
$$

for $\epsilon$ small enough and $c$ independent of $\epsilon$.

Proof. According to $(3.3),(3.5),(3.6)$, we have the following expansion in the $C^{2}$ norm as $\epsilon$ tends to 0 :

$$
\left|\phi_{\epsilon}^{\prime}\left(e^{i \theta}\right)\right|=\left|1+\epsilon R^{\prime}\left(e^{i \theta}\right)+\epsilon^{2} T_{\epsilon}^{\prime}\left(e^{i \theta}\right)\right|=1+\epsilon g_{1}(\theta)+O\left(\epsilon^{2}\right) .
$$

Since

$$
\mathcal{C}\left(\phi_{\epsilon}\left(e^{i \theta}\right)\right)=\left[1+\Re\left(\frac{\phi_{\epsilon}^{\prime \prime}\left(e^{i \theta}\right) e^{i \theta}}{\phi_{\epsilon}^{\prime}\left(e^{i \theta}\right)}\right)\right] /\left|\phi_{\epsilon}^{\prime}\left(e^{i \theta}\right)\right|,
$$

we also have (for the $C^{1}$-norm)

$$
\begin{aligned}
& \mathcal{C}\left(\phi_{\epsilon}\left(e^{i \theta}\right)\right)=1+\epsilon g_{2}(\theta)+O\left(\epsilon^{2}\right), \\
& \mathcal{C}_{M}=1+\epsilon g_{2}^{\infty}+O\left(\epsilon^{2}\right) \quad\left(g_{2}^{\infty}=\max g_{2}\right), \\
& \beta^{2}=2\left(\Lambda-\tau-\tau \epsilon g_{2}\right)+O\left(\epsilon^{2}\right)=2 \tau \epsilon\left(g_{2}^{\infty}-g_{2}\right)+O\left(\epsilon^{2}\right), \\
& (2 \Lambda-\tau \mathcal{C}) \mathcal{C}=\tau\left(1+2 \epsilon g_{2}^{\infty}\right)+O\left(\epsilon^{2}\right) .
\end{aligned}
$$

By replacing in (5.7), we obtain

$$
\mathcal{Q}(H)=\tau \int_{\Gamma_{0}} 2 \epsilon \ell H \mathcal{D}^{0}(\ell H)-\left(1+2 \epsilon g_{2}^{\infty}\right)\left(1+\epsilon g_{1}\right) H^{2}+{H^{\prime}}^{2}\left(1-\epsilon g_{1}\right)+\tilde{r}_{\epsilon}(H),
$$

where $\tilde{r}_{\epsilon}(H) \leq C \epsilon^{2}\|H\|_{H^{1}\left(\Gamma_{0}\right)}^{2}$. For this last estimate, we use, in particular, the fact that (see e.g. [4])

$$
\|u v\|_{H^{1 / 2}\left(\Gamma_{0}\right)} \leq k\|u\|_{H^{1 / 2}\left(\Gamma_{0}\right)}\|v\|_{C^{1}\left(\Gamma_{0}\right)} .
$$

Lemma 5.3 follows.

LEMMA 5.4. Assume $\Lambda=\tau \mathcal{C}_{M}$ and (3.8). Then there exists $\alpha>0$ independent of $\tau$ such that for $\epsilon$ small enough

$$
\begin{array}{ll}
\forall H \in Z_{0}^{1}\left(\Gamma_{0}\right) & \left.\mathcal{Q}_{1}+\epsilon \mathcal{Q}_{2}\right)(H) \geq \epsilon \alpha\|H\|_{H^{1}\left(\Gamma_{0}\right)}^{2}, \\
\forall H \in Z_{0}^{1}\left(\Gamma_{0}\right) & \mathcal{Q}(H) \geq \tau \epsilon \frac{\alpha}{2}\|H\|_{H^{1}\left(\Gamma_{0}\right)}^{2},
\end{array}
$$

$\mathcal{Q}$ is of type $(-1,+\infty)$ and the associated operator $\mathcal{A}$ is invertible. 
Proof. To prove (5.14), we apply Lemma 5.1 to $\mathcal{Q}_{1}+\epsilon \mathcal{Q}_{2}$ with

$$
\begin{aligned}
& \mathcal{V}=Z_{0}^{1}\left(\Gamma_{0}\right)=\left\{H \in H^{1}\left(\Gamma_{0}\right): \int_{\Gamma_{0}} H=0\right\} \\
& \mathcal{V}_{2}=\{\cos \theta, \sin \theta\} \text { the subspace of } \mathcal{V} \text { spanned by } \cos \theta, \sin \theta \\
& \mathcal{V}_{1}=\{\cos n \theta, \sin n \theta ; n \geq 2\} \text { the } L^{2} \text {-orthogonal of } \mathcal{V}_{2} \text { in } \mathcal{V}
\end{aligned}
$$

Then

$$
\phi_{1}(\cos n \theta, \cos \theta)=\int_{\Gamma_{0}} n \sin \theta \sin n \theta-\cos \theta \cos n \theta=0 \text { if } n \geq 2
$$

and similarly

$$
\phi_{1}(\cos n \theta, \sin \theta)=\phi_{1}(\sin n \theta, \cos \theta)=\phi_{1}(\sin n \theta, \sin \theta)=0 \text { if } n \geq 2,
$$

so that (5.1) is true. Obviously, by (5.10), $\mathcal{Q}_{1}$ vanishes on $\mathcal{V}_{2}$ so that (5.3) is true. Now if $v_{1} \in \mathcal{V}_{1}$, that is $v_{1}=\sum_{n \geq 2} a_{n} \cos n \theta+b_{n} \sin n \theta$, then

$$
\mathcal{Q}_{1}\left(v_{1}\right)=\pi \sum_{n \geq 2}\left(n^{2}-1\right)\left(a_{n}^{2}+b_{n}^{2}\right) \geq \pi \sum_{n \geq 2} \frac{n^{2}}{2}\left(a_{n}^{2}+b_{n}^{2}\right) \geq \alpha_{1}\left\|v_{1}\right\|_{H^{1}\left(\Gamma_{0}\right)}^{2},
$$

so that (5.2) is checked. Finally, if $v_{2}=\lambda \cos \theta+\mu \sin \theta$, we can write

$$
\mathcal{Q}_{2}\left(v_{2}\right)=\lambda^{2} a+2 \lambda \mu c+\mu^{2} b
$$

where

$$
a=\mathcal{Q}_{2}(\cos \theta), b=\mathcal{Q}_{2}(\sin \theta), c=\phi_{2}(\cos \theta, \sin \theta) .
$$

By (5.11), this numbers are given by the expressions (3.7). Therefore, if assumption (3.8) in Theorem 3.1 holds, the restriction of $\mathcal{Q}_{2}$ to $\mathcal{V}_{2}$ satisfies (5.4). The conditions of Lemma 5.1 being satisfied, we can deduce (5.14) (see (5.5)). Coupled with (5.9) and (5.12), we deduce (5.15) for $\epsilon$ small enough.

To obtain (5.16), recall that $\mathcal{V}=Z_{0}^{1}\left(\Gamma_{0}\right)$ is of codimension 1 in $\mathcal{H}=H^{1}\left(\Gamma_{0}\right)$. Then, since $\mathcal{Q}_{1}(1)=-2 \pi$, obviously, for $\epsilon$ small enough, $\mathcal{Q}(1)<0$. Coupled with (5.15), we deduce that the conditions of Lemma 4.3 are satisfied, whence (5.16).

Proof of Theorem 3.1. We use the same notations as in Lemma 5.4 (subspaces $\mathcal{V}, \mathcal{V}_{1}, \mathcal{V}_{2}$, forms $\mathcal{Q}_{1}, \mathcal{Q}_{2}$ ). Let us first prove the second part of the theorem. If (3.9) holds, then there exists $v_{2} \in \mathcal{V}_{2}$ such that $Q_{2}\left(v_{2}\right)<0$ (see (5.18)). Now we set $H_{\epsilon}=\lambda_{\epsilon}+v_{2}$ where $\lambda_{\epsilon}$ is chosen so that

$$
0=\int_{\Gamma_{0}} H_{\epsilon}\left|\phi_{\epsilon}^{\prime}\right|=\lambda_{\epsilon} \int_{\Gamma_{0}}\left|\phi_{\epsilon}^{\prime}\right|+\int_{\Gamma_{0}} v_{2}\left|\phi_{\epsilon}^{\prime}\right|
$$

Since $\int_{\Gamma_{0}} v_{2}=0$, by (5.13) and the fact that $\int_{\Gamma_{0}}\left|\phi_{\epsilon}^{\prime}\right| \rightarrow 2 \pi$ as $\epsilon \rightarrow 0$, we deduce

$$
\left|\lambda_{\epsilon}\right|=O(\epsilon) \text {. }
$$

Now

$$
\left(\mathcal{Q}_{1}+\epsilon \mathcal{Q}_{2}\right)\left(\lambda_{\epsilon}+v_{2}\right)=\lambda_{\epsilon}^{2}\left(\mathcal{Q}_{1}+\epsilon \mathcal{Q}_{2}\right)(1)+2 \lambda_{\epsilon}\left(\phi_{1}+\epsilon \phi_{2}\right)\left(1, v_{2}\right)+\left(\mathcal{Q}_{1}+\epsilon \mathcal{Q}_{2}\right)\left(v_{2}\right) .
$$


Since $\phi_{1}\left(1, v_{2}\right)=0$ and $\mathcal{Q}_{1}\left(v_{2}\right)=0$, by $(5.20)$

$$
\left(\mathcal{Q}_{1}+\epsilon \mathcal{Q}_{2}\right)\left(\lambda_{\epsilon}+v_{2}\right)=\epsilon \mathcal{Q}_{2}\left(v_{2}\right)+o(\epsilon)
$$

This can be made negative for $\epsilon$ small enough since $\mathcal{Q}_{2}\left(v_{2}\right)<0$. Therefore if $h_{\epsilon}\left(\phi_{\epsilon}\left(e^{i \theta}\right)\right)=H_{\epsilon}(\theta)$, we have by $(5.9),(5.12)$ and $(5.19)$

$$
\int_{\Gamma *} h_{\epsilon}=0 \quad, \quad Q\left(h_{\epsilon}\right)<0 \quad \text { for } \epsilon \text { small enough. }
$$

We now come to the first part of the theorem. Assume (3.8) holds. According to Lemma 5.2, we have to prove that the restriction of $\mathcal{Q}$ to the subspace of $H \in H^{1}\left(\Gamma_{0}\right)$ such that $\int_{\Gamma_{0}} H\left|\phi_{\epsilon}^{\prime}\right|=0$ is $H^{1}\left(\Gamma_{0}\right)$-coercive (note that, since $H=h \circ \Phi_{\epsilon}$, by (5.13), there exists $C$ such that for $\epsilon$ small, $\left.\|h\|_{H^{1}\left(\Gamma^{*}\right)}^{2} \leq C\|H\|_{H^{1}\left(\Gamma_{0}\right)}\right)$. Note that

$$
\int_{\Gamma_{0}} H\left|\phi_{\epsilon}^{\prime}\right|=<\left|\phi_{\epsilon}^{\prime}\right|, H>_{H^{-1}\left(\Gamma_{0}\right) \times H^{1}\left(\Gamma_{0}\right)}=<\mathcal{R}^{-1}\left(\left|\phi_{\epsilon}^{\prime}\right|\right), H>_{H^{1}\left(\Gamma_{0}\right) \times H^{1}\left(\Gamma_{0}\right)},
$$

so that

$$
\left\{H \in \mathcal{H} ; \int_{\Gamma_{0}} H\left|\phi_{\epsilon}^{\prime}\right|=0\right\}=\left[\mathcal{R}^{-1}\left(\left|\phi_{\epsilon}^{\prime}\right|\right)\right]^{\perp} .
$$

By Lemma 5.4, for $\epsilon$ small enough, $\mathcal{Q}$ is of type $(-1,+\infty)$ and coercive on $Z_{0}^{1}\left(\Gamma_{0}\right)$. If we write

$$
\mathcal{Q}(H)=\tau<A_{\epsilon} H, H>_{H^{-1}}\left(\Gamma_{0}\right) \times H^{1}\left(\Gamma_{0}\right),
$$

by Theorem 4.4, to complete the proof of the theorem, we only have to prove that

$$
0>\mathcal{Q}\left(A_{\epsilon}^{-1}\left(\left|\phi_{\epsilon}^{\prime}\right|\right)\right)=\tau<\left|\phi_{\epsilon}^{\prime}\right|, A_{\epsilon}^{-1}\left|\phi_{\epsilon}^{\prime}\right|>_{H^{-1} \times H^{1}} .
$$

We now denote by $C$ all positive constants independent of $\epsilon$. We have from $(5.9-5.13)$ and $(5.21)$

$$
\left|\phi_{\epsilon}^{\prime}\right|=1+\epsilon g_{\epsilon} \quad, \quad\left\|g_{\epsilon}\right\|_{H^{1}\left(\Gamma_{0}\right)} \leq C, A_{\epsilon} H=A_{1} H+\epsilon \widetilde{A}_{\epsilon} H
$$

where $A_{1} H=-H-H^{\prime \prime}, \quad\left\|\widetilde{A}_{\epsilon} H\right\|_{H^{-1}\left(\Gamma_{0}\right)} \leq C\|H\|_{H^{1}\left(\Gamma_{0}\right)}$.

Now, we set $\omega_{\epsilon}=A_{\epsilon}^{-1}\left(\left|\phi_{\epsilon}^{\prime}\right|\right)$ and we write $\omega_{\epsilon}=\lambda_{\epsilon}+\sigma_{\epsilon}+\tau_{\epsilon}$, the decomposition of $\omega_{\epsilon}$ along $\{1\} \oplus \mathcal{V}_{2} \oplus \mathcal{V}_{1}$ (see Lemma 5.4). According to (5.22), we need to prove that $\int_{\Gamma_{0}} \omega_{\epsilon}\left|\phi_{\epsilon}^{\prime}\right|<0$ for $\epsilon$ small enough. Here, $\left|\phi_{\epsilon}^{\prime}\right|$ tends to 1 but, $A_{\epsilon}$ tends to $A_{1}$ which is not invertible. Therefore, we need to control carefully each term in the decomposition of $\omega_{\epsilon}$.

By definition of $\omega_{\epsilon},\left|\phi_{\epsilon}^{\prime}\right|=A_{\epsilon} \omega_{\epsilon}$, that is, using $A_{1} 1=-1, A_{1} \sigma_{\epsilon}=0$ :

$$
1+\epsilon g_{\epsilon}=-\lambda_{\epsilon}+A_{1} \tau_{\epsilon}+\epsilon \widetilde{A}_{\epsilon} \omega_{\epsilon} .
$$

Integrating over $\Gamma_{0}$ gives (since $\int_{\Gamma_{0}} A_{1} \tau_{\epsilon}=0$ )

$$
2 \pi=-2 \pi \lambda_{\epsilon}+\epsilon \int_{\Gamma_{0}}-g_{\epsilon}+\widetilde{A}_{\epsilon}\left(\lambda_{\epsilon}+\sigma_{\epsilon}+\tau_{\epsilon}\right) .
$$

From this, we will only keep in mind that

$$
\left|1+\lambda_{\epsilon}\right| \leq C \epsilon\left(1+\left\|\sigma_{\epsilon}\right\|_{H^{1}\left(\Gamma_{0}\right)}+\left\|\tau_{\epsilon}\right\|_{H^{1}\left(\Gamma_{0}\right)}\right) .
$$


Multiplying (5.23) by $\tau_{\epsilon}$ and integrating give, since $\int_{\Gamma_{0}} \tau_{\epsilon}=0$

$$
\epsilon \int_{\Gamma_{0}} g_{\epsilon} \tau_{\epsilon}=<A_{1} \tau_{\epsilon}, \tau_{\epsilon}>+\epsilon<\widetilde{A}_{\epsilon}\left(\lambda_{\epsilon}+\sigma_{\epsilon}+\tau_{\epsilon}\right), \tau_{\epsilon}>.
$$

We use the coercivity of $A_{1}$ on $\mathcal{V}_{1}$ (see (5.17)) to deduce from (5.25)

$$
\alpha_{1}\left\|\tau_{\epsilon}\right\|_{H^{1}\left(\Gamma_{0}\right)}^{2} \leq C \epsilon|| \tau_{\epsilon} \|_{H^{1}\left(\Gamma_{0}\right)}\left(1+\left|\lambda_{\epsilon}\right|+\left\|\sigma_{\epsilon}\right\|_{H^{1}\left(\Gamma_{0}\right)}+\left\|\tau_{\epsilon}\right\|_{H^{1}\left(\Gamma_{0}\right)}\right),
$$

which implies also for $C \epsilon \leq \frac{\alpha_{1}}{2}$

$$
\frac{\alpha_{1}}{2}|| \tau_{\epsilon} \|_{H^{1}\left(\Gamma_{0}\right)} \leq C \epsilon\left(1+\left|\lambda_{\epsilon}\right|+\left\|\sigma_{\epsilon}\right\|_{H^{1}\left(\Gamma_{0}\right)}\right) .
$$

Next we rewrite (5.23) as

$$
1+\epsilon g_{\epsilon}=-\lambda_{\epsilon}+\epsilon \widetilde{A}_{\epsilon}\left(\lambda_{\epsilon}\right)+A_{\epsilon} \sigma_{\epsilon}+A_{1} \tau_{\epsilon}+\epsilon \widetilde{A}_{\epsilon}\left(\tau_{\epsilon}\right)
$$

and we multiply it by $\sigma_{\epsilon}$ and integrate to obtain

$$
\epsilon \int_{\Gamma_{0}} g_{\epsilon} \sigma_{\epsilon}=\int_{\Gamma_{0}} \epsilon \lambda_{\epsilon} \sigma_{\epsilon} \widetilde{A}_{\epsilon}(1)+\epsilon \sigma_{\epsilon} \widetilde{A}_{\epsilon} \tau_{\epsilon}+\sigma_{\epsilon} A_{\epsilon} \sigma_{\epsilon} .
$$

Under the assumption (3.8), we have by (5.15)

$$
\frac{1}{\tau} \mathcal{Q}\left(\sigma_{\epsilon}\right)=\int_{\Gamma_{0}} \sigma_{\epsilon} A_{\epsilon} \sigma_{\epsilon} \geq \epsilon \alpha\left\|\sigma_{\epsilon}\right\|_{H^{1}\left(\Gamma_{0}\right)}^{2} \quad \text { for some } \alpha>0 .
$$

We deduce from (5.27), (5.28)

$$
\epsilon \alpha\left\|\sigma_{\epsilon}\right\|_{H^{1}\left(\Gamma_{0}\right)}^{2} \leq C \epsilon|| \sigma_{\epsilon} \|_{H^{1}\left(\Gamma_{0}\right)}\left(1+\left|\lambda_{\epsilon}\right|+\left\|\tau_{\epsilon}\right\|_{H^{1}\left(\Gamma_{0}\right)}\right) .
$$

We simplify by $\epsilon\left\|\sigma_{\epsilon}\right\|_{H^{1}\left(\Gamma_{0}\right)}$ and use (5.26) to obtain for $\epsilon$ small enough (and some other C)

$$
\left.\left\|\sigma_{\epsilon}\right\|_{H^{1}\left(\Gamma_{0}\right)} \leq C\left(1+\left|\lambda_{\epsilon}\right|\right), \quad\left\|\tau_{\epsilon}\right\|_{H^{1}\left(\Gamma_{0}\right)}\right) \leq C \epsilon\left(1+\left|\lambda_{\epsilon}\right|\right) .
$$

We now go back to (5.24) and with the help of (5.29), we prove

$$
\left|1+\lambda_{\epsilon}\right| \leq C \epsilon\left(1+\left|\lambda_{\epsilon}\right|\right),
$$

which implies that $\left|\lambda_{\epsilon}\right|$ is bounded for $\epsilon$ small enough and $\lim _{\epsilon \rightarrow 0}\left|1+\lambda_{\epsilon}\right|=0$. Finally, we can control the expression in (5.22), namely

$$
\mathcal{Q}\left(\omega_{\epsilon}\right) / \tau=\int_{\Gamma_{0}} \omega_{\epsilon}\left|\phi_{\epsilon}^{\prime}\right|=\int_{\Gamma_{0}}\left(\lambda_{\epsilon}+\sigma_{\epsilon}+\tau_{\epsilon}\right)\left(1+\epsilon g_{\epsilon}\right)=2 \pi \lambda_{\epsilon}+\epsilon \int_{\Gamma_{0}} g_{\epsilon}\left(\lambda_{\epsilon}+\sigma_{\epsilon}+\tau_{\epsilon}\right) .
$$

and

$$
\left|\mathcal{Q}\left(\omega_{\epsilon}\right)+2 \pi \tau\right| \leq 2 \pi \tau\left|1+\lambda_{\epsilon}\right|+C \epsilon\left(1+\left|\lambda_{\epsilon}\right|\right),
$$

so that $\lim _{\epsilon \rightarrow 0} \mathcal{Q}\left(\omega_{\epsilon}\right)=-2 \pi \tau<0$, whence (5.22). 
Proof of Corollary 3.3. We easily compute the necessary quantities and, in particular

$$
\begin{gathered}
g_{1}(\theta)=-(2 k+1) \cos (2 k+2) \theta, g_{2}=-(2 k+3) g_{1}, g_{2}^{\infty}=(2 k+1)(2 k+3), \\
\ell(\theta)=\sqrt{2 g_{2}^{\infty}} \sin (k+1) \theta, \\
a=b=2 k(2 k+1)(2 k+3) \pi \quad \text { and } \quad c=0 .
\end{gathered}
$$

The conclusion of the corollary follows by applying Theorem 3.1.

REMARK 5.5. In the case $k=0$ where $\Gamma^{*}$ is an ellipse, the restriction of $\mathcal{Q}_{2}$ to $\mathcal{V}_{2}$ is identically zero. Therefore, it is necessary to pursue the expansion with respect to $\epsilon$ a little further to conclude.

Proof of Corollary 3.5. By the computations in [15], if $f$ is the sum of $N$ Dirac masses, then, for $\tau$ large, $\Gamma^{*}$ is close to a circle and the constants $a, b, c$ of Theorem 3.1 write

$$
\begin{gathered}
a=2 \pi \sum_{1 \leq k, j \leq N} \alpha_{k} \alpha_{j}\left[\frac{\left(1+\bar{z}_{k}^{2}\right)\left(1+z_{j}^{2}\right)}{\left(z_{j} \bar{z}_{k}-1\right)^{2}}-2 \frac{1}{z_{j} \bar{z}_{k}-1}\right], \\
b=2 \pi \sum_{1 \leq k, j \leq N} \alpha_{k} \alpha_{j}\left[\frac{\left(1-\bar{z}_{k}^{2}\right)\left(1-z_{j}^{2}\right)}{\left(z_{j} \bar{z}_{k}-1\right)^{2}}-2 \frac{1}{z_{j} \bar{z}_{k}-1}\right], \\
c=-2 \pi \frac{1}{i} \sum_{1 \leq k, j \leq N} \alpha_{k} \alpha_{j} \frac{\bar{z}_{k}^{2}-z_{j}^{2}}{\left(z_{j} \bar{z}_{k}-1\right)^{2}},
\end{gathered}
$$

where, up to a global constant, the $\alpha_{i}$ 's are the coefficients of the given Dirac masses and where the $z_{i}$ are their location. We choose $N=4, \alpha_{k}=(-1)^{k}$ for $1 \leq k \leq 4, z_{2 p-1}=(-1)^{p+1}(\alpha+i \beta), z_{2 p}=(-1)^{p+1}(-\alpha+i \beta)$ for $p=1,2$. By symmetry $c=0$. Then, explicit computations show that there exists a region in the set $\{(\alpha, \beta) ; \alpha, \beta>0, \alpha \neq \beta\}$ where $a<0, b>0$. The conclusion follows from Theorem 3.1.

Proof of Theorem 3.\%. We recall that

$$
Q(h)=\int_{\Gamma^{*}} \beta h \mathcal{D}(\beta h)-(2 \Lambda-\tau \mathcal{C}) \mathcal{C} h^{2}+\tau h^{\prime 2} .
$$

If $\tau=0, Q(h)=Q_{0}(h)=2 \Lambda \int_{\Gamma^{*}} h \mathcal{D} h-\mathcal{C} h^{2}$. Moreover, we may write, for all $\tau \in\left(0, \Lambda / 2 \mathcal{C}_{M}\right)$

$$
Q(h)=Q_{0}(h)+\tau Q_{\tau}(h), \text { with }\left|Q_{\tau}(h)\right| \leq k\|h\|_{H^{1}\left(\Gamma^{*}\right)}^{2},
$$

where $k=k\left(\Lambda, \Gamma^{*}\right)$. It is proved in ([9]) that, if $\Gamma^{*}$ is different from a circle, then

$$
\exists h \in H^{1}\left(\Gamma^{*}\right) \text { such that } Q_{0}(h)<0, \int_{\Gamma^{*}} h=0,\|h\|_{H^{1}\left(\Gamma^{*}\right)} \leq 1 .
$$


Let $\tau_{0}=\min \left\{-Q_{0}(h) / k, \Lambda / 2 \mathcal{C}_{M}\right)$. It follows that, for $\tau \in\left(0, \tau_{0}\right)$, $Q(h) \leq Q_{0}(h)+k \tau<0$. This proves the theorem.

Proof of Theorem 3.10. It is similar to the proof of the second part of Theorem 3.1. Expanding

$$
\beta=\sqrt{2(\Lambda-\tau \mathcal{C})}=\sqrt{2(\Lambda-\tau)}\left(1-\frac{\epsilon \tau g_{2}}{2(\Lambda-\tau)}+O\left(\epsilon^{2}\right)\right)
$$

we write

$$
\mathcal{Q}=\widehat{\mathcal{Q}}_{1}+\epsilon \widehat{\mathcal{Q}}_{2}+\widehat{r}_{\epsilon}
$$

where

$$
\begin{gathered}
\widehat{\mathcal{Q}}_{1}(H)=\int_{\Gamma_{0}} 2(\Lambda-\tau) H \mathcal{D}^{0}(H)-(2 \Lambda-\tau) H^{2}+\tau H^{\prime 2}, \\
\widehat{\mathcal{Q}}_{2}(H)=-\int_{\Gamma_{0}} \tau\left[H \mathcal{D}^{0}\left(g_{2} H\right)+g_{2} H \mathcal{D}^{0}(H)\right]+\left[(2 \Lambda-\tau) g_{1}+2(\Lambda-\tau) g_{2}\right] H^{2}+\tau g_{1} H^{\prime 2},
\end{gathered}
$$

and

$$
\left|\widehat{r}_{\epsilon}(H)\right| \leq c \epsilon^{2}\|H\|_{H^{1}\left(\Gamma_{0}\right)}^{2} .
$$

We check that $\hat{\mathcal{Q}}_{1}$ vanishes on $\mathcal{V}_{2}$ and that $\mathcal{V}_{1}$ and $\mathcal{V}_{2}$ are orthogonal with respect to $\phi_{1}(\cdot, \cdot)$. Now, if $v_{2}=\lambda \cos \theta+\mu \sin \theta$, then $\widehat{\mathcal{Q}}_{2}\left(v_{2}\right)=\lambda^{2} \widehat{a}+2 \lambda \mu \widehat{c}+\mu^{2} \widehat{b}$,

where $\widehat{a}=\widehat{\mathcal{Q}}_{2}(\cos \theta), \widehat{b}=\widehat{\mathcal{Q}}_{2}(\sin \theta), \widehat{c}=\widehat{\phi}_{2}(\cos \theta, \sin \theta)$. We check that $\widehat{a}, \widehat{c}$ are given by the expressions (3.10) and that $\widehat{b}=-\widehat{a}$. By assumption, they are not all equal to 0 so that $\widehat{c}^{2}-\widehat{a} \widehat{b}>0$ and we can find $v_{2} \in \mathcal{V}_{2}$ such that $\widehat{\mathcal{Q}}_{2}\left(v_{2}\right)<0$. Then, we finish as in the proof of the second part of Theorem 3.1.

Proof of corollary 3.12. By (3.10), the computation of $\widehat{a}, \widehat{b}, \widehat{c}$ gives

$$
\widehat{a}=-\widehat{b}=-(2 \Lambda+\tau) \pi \sigma_{1} \quad \text { and } \quad \widehat{c}=0
$$

whence (3.11) if $\sigma_{1} \neq 0$. Then, we apply Theorem 3.10.

6. Appendix: proof of Proposition 2.2. The result of Proposition 2.2 is obtained by differentiating each of the three terms in the total energy (1.2). Most of the necessary computations are available in [21]. A difference is that everything is stated in [21] for bounded domains while here we consider exterior domains. However, the main point behind the structure theorem which is reproduced here in Lemma 2.1, is based on the fact that any regular small perturbation of a regular domain may be represented through normal displacements to the boundary. This is true whether we consider perturbations of the domain itself or of its complement. Therefore, Lemma 2.1 may be stated without any change. Moreover, the computations for each of the three energy terms involved here are exactly the same, except may be for the signs (we have to switch from exterior to interior normal derivatives in some places). 
According to Lemma 2.1, let us denote by $l_{i}^{S}, l_{i}^{P}, l_{i}^{M}, i=1,2$ the linear and bilinear forms respectively describing the first and second derivatives of each of the functionals

$$
\Omega \rightarrow S\left({ }^{c} \Omega\right), P(\Omega), \int_{\Omega}\left|\nabla u_{\Omega}\right|^{2},
$$

where $u_{\Omega}$ is solution of the exterior problem (1.1). Note that, by (1.2), if $l_{1}, l_{2}$ denote the forms associated with the global functional $E(\cdot)$, we have for $i=1,2$

$$
l_{i}=-\frac{1}{2} l_{i}^{M}+\tau l_{i}^{P}-\Lambda l_{i}^{S} .
$$

The derivatives of the three terms are computed in [21] for bounded domains. The results may be applied directly here. Indeed, the perimeter of a set is the same as the perimeter of its complement. The derivative of $\theta \rightarrow S\left({ }^{c}(I+\theta)(\Omega)\right)$ is the same except that the interior normal derivative to $\partial \Omega^{*}$ is involved rather than the exterior one. Finally, for the Dirichlet problem (1.1), the computations are exactly the same, up to the signs at the boundary.

Recall that, here, we have denoted by $\nu$ the interior unit normal derivative to $\Gamma^{*}=\partial \Omega^{*}$, which is directed toward $\Omega^{*}$, and by $\mathcal{C}$ the curvature of $\Gamma^{*}$ seen from outside $\Omega^{*}$ (for instance, it is positive if $\Gamma^{*}$ is a circle). Recall also that displacements are positive when they are made in the direction of $\nu$, that is toward the inside of $\Omega^{*}$. We have (see [21]):

$$
\begin{gathered}
l_{1}^{S}(h)=\int_{\Gamma^{*}} h, l_{2}^{S}(h, h)=\int_{\Gamma^{*}} \mathcal{C} h^{2}, \\
l_{1}^{P}(h)=\int_{\Gamma^{*}} \mathcal{C} h, l_{2}^{P}(h, h)=\int_{\Gamma^{*}}\left|\nabla^{\tau} h\right|^{2}
\end{gathered}
$$

where $\nabla^{\tau}$ denotes the tangential gradient,

$$
l_{1}^{M}(h)=-\int_{\Gamma^{*}}|\nabla u|^{2} h, \quad l_{2}^{M}(h, h)=\int_{\Gamma^{*}} 2 \omega(h) \frac{\partial \omega(h)}{\partial \nu}-h^{2}\left[2 \frac{\partial u}{\partial \nu} \frac{\partial^{2} u}{\partial \nu^{2}}+\mathcal{C}\left(\frac{\partial u}{\partial \nu}\right)^{2}\right],
$$

where $\omega(h)$ is the solution of

$$
\Delta \omega(h)=0 \text { in } \Omega, \omega(h)=-h \frac{\partial u}{\partial \nu} \text { on } \Gamma,|\nabla \omega(h)| \rightarrow 0 \text { at } \infty .
$$

We easily check that $\nabla^{\tau} h=h^{\prime}$, where the derivative is taken with respect to the length parameter, and that $\omega(h) \frac{\partial \omega(h)}{\partial \nu}=-h \beta \mathcal{D}(h \beta)$ (recall that $\beta=\partial u / \partial \nu$ ). Next a simple local computation (see e.g.[17],[9]) gives

$$
\frac{\partial u}{\partial \nu} \frac{\partial^{2} u}{\partial \nu^{2}}=-\mathcal{C} \beta^{2}-f \beta
$$

Now the expressions of the forms $l_{1}, l_{2}$ associated with the derivatives of $E(\cdot)$ follow $(\operatorname{see}(6.1))$ :

$$
l_{1}(h)=\int_{\Gamma^{*}}\left(\frac{1}{2} \beta^{2}+\tau \mathcal{C}-\Lambda\right) h
$$




$$
l_{2}(h, h)=\int_{\Gamma^{*}} \beta h D(\beta h)-\left(\mathcal{C} \beta^{2} / 2+\Lambda \mathcal{C}+f \beta\right) h^{2}+\tau{h^{\prime}}^{2} .
$$

If $\Omega^{*}$ is an equilibrium shape, that is $l_{1} \equiv 0$, we have $\beta^{2}=2(\Lambda-\tau \mathcal{C})$. We plug this identity into the above expression of $l_{2}(h, h)$ to obtain $(2.6)$.

\section{REFERENCES}

[1] S. Belov AND N. FujI, Symmetry and sufficient condition of optimality in a domain optimization problem, Control and Cybernetics, $26: 1$ (1997), pp. 45-56.

[2] D. Bucur and J.P. Zolésio, Anatomy of the Shape Hessian Via Lie Brackets, Ann. Mat. Pura Appl., (IV) CLXXIII (1997), pp. 127-143.

[3] M. CrouzeIX, Variational approach of a magnetic shaping problem, Eur. J. Mech. B Fluids, 10 (1991), pp. 527-536.

[4] M. Dambrine, On variations of the shape Hessian and sufficient conditions for the stability of critical shapes, Rev. R. Acad. Cien. Serie A. Mat., 96:1 (2002), pp. 95-121.

[5] M. Dambrine And M. Pierre, About stability of equilibrium shapes, M2AN, 34:4 (2000), pp. 811-834.

[6] Dautray and J.L. Lions, Analyse mathématique et Calcul numérique pour les sciences et les techniques, Vol.1, Masson, 1984.

[7] M. Delfour and J. P. Zolésio, Velocity Method and Lagrangian Formulation for the Computation of the Shape Hessian, SIAM Control Optim., 29 (1991), pp. 513-526.

[8] J. Descloux, A stability result for the magnetic shaping problem, Z. Angew. Math. Phys., 45:4 (1994), pp. 543-555.

[9] J. Descloux, Stability of the solutions of the bidimensional magnetic shaping problem in absence of surface tension, Eur. J. Mech, B/Fluids, 10 (1991), pp. 513-526.

[10] K. EpPLER, Second derivatives and sufficient optimality conditions for shape functionals, Control Cybern., 29:2 (2000), pp. 485-511.

[11] N. FuJI, Sufficient conditions for optimality in shape optimization, Control Cybern., 23 (1994), pp. 393-406.

[12] D. Gilbarg and N.S Trudinger, Elliptic Partial Differential Equations of Second order, Springer-Verlag, Berlin, 1983.

[13] J. Hadamard, Mémoire sur le problème d'analyse relatif à l'équilibre des plaques élastiques encastrées (1908), Oeuvres de J. Hadamard, CNRS Paris, 1968.

[14] A. Henrot And M. Pierre, Un problème inverse en formage de métaux liquides, RAIRO Modél. Math. Anal. Num., 23 (1989), pp. 155-177.

[15] A. Henrot and M. Pierre, About Existence of equilibria in Electromagnetic Casting, Quarterly of Applied Math., Vol. XLIX, Nr 3 (1991), pp. 563-575.

[16] A. Henrot and M. Pierre, About Critical Points of the Energy in an Electromagnetic Shaping Problem, Lect. Notes in Control and Inf., Nr 178, J.P. Zolézio ed. (1992), pp. 238-253.

[17] A. Henrot, M. Pierre and M. Rihani, Positivity of the shape Hessian and instability of some equilibrium shapes, to appear in Mediterr. J. Math.

[18] J.H. Maddocks, Stability and folds, Arch. Rat. Mech. Anal., 99 (1987), pp. 301-328.

[19] J.H. MADDOCKS, Restricted quadratic forms and their application to bifurcation and stability in constrained variational principles, SIAM J. Math. Anal., 16 (1985), pp. 47-68.

[20] F. Murat and J. Simon, Sur le contrôle par un domaine géométrique, Rapport L. A. 189, Univ. Paris 6, France (1976).

[21] A. Novruzi And M. Pierre, Structure of shape derivatives, J. of Evolution Equations, 2 (2002), pp. 365-382.

[22] J. Simon, Differentiation with respect to the domain in boundary value problems, Num. Funct. Ana. Opt., 2 (1980), pp. 649-687.

[23] J. SOKOLOWSKI AND J. P. ZolÉZIO, Introduction to shape optimization, Springer-Verlag, Berlin (1992). 